\title{
Unsupervised mixture-eliminating estimation of equivalent number of looks for PolSAR data
}

\author{
Dingsheng Hu, Student Member, IEEE, Stian Normann Anfinsen, Member, IEEE, Xiaolan Qiu, Member, IEEE, \\ Anthony Paul Doulgeris, Member, IEEE, and Bin Lei, Member, IEEE,
}

\begin{abstract}
This paper addresses the impact of mixtures between classes on equivalent number of looks (ENL) estimation. We propose an unsupervised ENL estimator for polarimetric synthetic aperture radar (PolSAR) data, which is based on small sample estimates but incorporates a mixture-eliminating procedure to automatically assess the uniformity of the estimation windows. A statistical feature derived from a combination of linear and logarithmic moments is investigated and adopted in the procedure, as it has different mean values for samples from uniform and non-uniform windows. We introduce an approach to extract the approximated sampling distribution of this test statistic for uniform windows. Then the detection is conducted by a hypothesis test with adaptive thresholds determined by a nonuniformity ratio. Finally the experiments are performed on both simulated and real SAR data. The capability of the unsupervised mixture-eliminating procedure is verified with simulated data. In the real-data experiments, the ENL estimates of Flevoland and San Francisco PolSAR images are analyzed, which show the robustness of the proposed ENL estimation for SAR scenes with different complexities.
\end{abstract}

Index Terms-Equivalent number of looks (ENL) estimation, uniform window, automatic processing chain, log-cumulant, polarimetric Synthetic Aperture Radar (SAR).

\section{INTRODUCTION}

$\mathbf{T}$ HE equivalent number of looks (ENL) is an important parameter of multilook synthetic aperture radar (SAR) images. In the multilooking process, the SAR measurements are averaged to mitigate the noise-like effect of interference, known as speckle, which is a feature of all coherent imaging systems. As the averaged measurements are correlated, it is complicate to statistically model the output data. The ENL is defined to replace the actual number of correlated samples by an equivalent number of independent ones, thus the multilook data can be modeled as an average of a fractional number of independent measurements.

Being a model parameter, the ENL has influence on the accuracy of the information extracted by methods based upon parametric models of multilook SAR data. For instance, the ENL is a necessary input to important classification [1][2][3]

This work was supported in part by the NSFC under Grant 61331017 and in part by the fund of the GF-3 High-Resolution Earth Observation System under Project 30-Y20A12-9004-15/16

Dingsheng Hu, Xiaolan Qiu and Bin Lei were with the Key Laboratory of Technology in Geo-spatial Information Processing and Application System in the Institute of Electronics, Chinese Academy of Sciences (IECAS), Beijing 100190, China e-mail: (xlqiu@mail.ie.ac.cn).

Dingsheng $\mathrm{Hu}$ was also with the University of Chinese Academy of Sciences (UCAS), Beijing 100049, China.

Stian Normann Anfinsen, and Anthony Paul Doulgeris were with Department of Physics and Technology, UiT The Arctic University of Norway, Tromsø 9037, Norway. and change detection algorithms [4] for multilook polarimetric SAR (PolSAR) data.

The ENL is commonly estimated by identifying homogeneous regions in an image, where speckle is fully developed and the sources of heterogeneity, like texture or mixtures of different classes, are assumed ignorable [5]. The selection of regions of interest is usually done manually. However, as the creation of fully automatic processing chains is a goal in many application of SAR data, these chains will obviously benefit from having a robust and automatic ENL estimation method.

Some attempts have already been made to design a fully automatic estimation algorithm that avoids manual selection of a region of interest. In an arbitrary SAR scene, there are normally a variety of land covers with homogeneous regions of various sizes scattered across the images. This motivates an idea, where the ENL estimator is evaluated in small windows over the whole images. The ENL is then inferred from the collection of small sample estimates. Based on this idea, several unsupervised estimation approaches have been proposed. The method used in [6][7] is to produce a scatter plot of mean versus standard deviation of the intensity data in each small window. The idea is that values computed under fully developed speckle will dominate the population of estimates and stand out as a linear feature. Then the ENL can be inferred from the slope. Another approach from [8] is to compute the ENL itself in the small windows, and then produce a one-dimensional distribution of small sample ENL estimates. Necessarily, a large enough proportion of the estimation windows are assumed to be homogeneous. Therefore the overall distribution of estimates should be dominated by estimates computed in windows that only contain variation due to fully developed speckle, and the mode value can be used as an estimate of the ENL. Papers [9] and [10] both follow this approach, but with different ENL estimators computed in the windows.

The estimators adopted in the latter papers are proposed to use the full sample polarimetric covariance matrix, which is a common representation of multilook PolSAR data, as input. In [9], two estimators are given by assuming the sample covariance matrix is complex Wishart distributed. One is based on second-order trace moments. Another is obtained from the log-determinant matrix moment and is also shown to be the maximum likelihood (ML) estimator under the Wishart model. The second estimator, or ML estimator for short, proves to have much lower variance than any other known ENL estimator [9][10]. But for heterogeneous regions, the ML method will produce underestimation. The other paper 
[10] introduces an estimator, named Development of Trace Moments (DTM), which uses trace moments to cancel effects of textural variation on the ENL estimation. It is claimed to be texture invariant under the product model.

However, within the framework of small sample ENL estimation, the presence of multiple classes in the estimation windows is still left out of consideration. Furthermore, there could be complex scenes where a majority of the small estimation windows contain a mixture of different classes. These windows would contribute low ENL values to the collection. Then, if we still take the mode value as the final estimate of the ENL, the unsupervised algorithm would definitely produce an underestimated global ENL. Therefore, it is necessary to find a novel and unsupervised estimator that can be used to estimate the accurate ENL even for a complicated SAR image containing a high proportion of fine scale class mixtures. These non-uniform windows, which contain multiple classes, are difficult to model statistically. To reduce their effect on the final estimate, it is desirable to detect these windows first and remove them from the collection before further analysis. Our former work [11] shows that in mixture areas, inconsistent ENL estimates will be obtained in different polarimetric channels. This preliminary observation gives us clue to a powerful multipolarization SAR descriptor for uniform windows detection.

This paper is organized as follows. Section II introduces the basic data formats and commonly used models for multilook PolSAR data. Section III presents the main characteristics of the proposed unsupervised ENL estimation chain. Section IV demonstrates the performance of our method in experiments with synthetic and real data. Finally, conclusions are given in Section V.

\section{Polarimetric SAR Image Model}

\section{A. Data Format}

A full-polarimetric imaging radar measures the amplitude and phase of backscattered signals in the four combinations of the linear receive and transmit polarizations: $\mathrm{HH}, \mathrm{HV}, \mathrm{VH}$, and VV. Assuming that the target reciprocity condition is satisfied [12], the backscattering of a monostatic polarimetric SAR system is characterized by the complex scattering vector, $\mathbf{s}$, with dimension $d=3$, as given by

$$
\mathbf{s}=\left[S_{H H}, \sqrt{2} S_{C r o s s}, S_{V V}\right]^{T}
$$

The elements represent the complex backscattering coefficients in three polarimetric channels, noting that $S_{\text {Cross }}$ is a coherent average of the $\mathrm{HV}$ and $\mathrm{VH}$ channels measurements. The superscript $T$ denotes the matrix transpose, and $\sqrt{2}$ arises from the requirement to conserve the total scattered power, after coherent averaging of the cross-polarization channels.

The scattering vector $\mathbf{s}$ is a single-look complex (SLC) format representation of PolSAR data. Single and dual-channel polarimetric data can be treated in a similar way, as subsets of lesser dimension. The scattering vectors are transformed into multilook sample covariance matrices in order to reduce speckle, i.e,

$$
\begin{aligned}
\mathbf{C} & =\frac{1}{L} \sum_{i=1}^{L} \mathbf{s}_{i} \mathbf{s}_{i}{ }^{H} \\
& =\left[\begin{array}{ccc}
\left\langle\left|S_{H H}\right|^{2}\right\rangle & \left\langle\sqrt{2} S_{H H} S_{\text {Cross }}^{*}\right\rangle & \left\langle S_{H H} S_{V V}^{*}\right\rangle \\
\left\langle\sqrt{2} S_{\text {Cross }} S_{H H}^{*}\right\rangle & \left\langle 2\left|S_{\text {Cross }}\right|^{2}\right\rangle & \left\langle\sqrt{2} S_{\text {Cross }} S_{V V}^{*}\right\rangle \\
\left\langle S_{V V} S_{H H}^{*}\right\rangle & \left\langle\sqrt{2} S_{V V} S_{\text {Cross }}^{*}\right\rangle & \left\langle\left|S_{V V}\right|^{2}\right\rangle
\end{array}\right]
\end{aligned}
$$

Here $L$ is the nominal number of looks used for averaging, $(\cdot)^{H}$ and $(\cdot)^{*}$ mean the Hermitian transposition operator and complex conjugation, respectively, and $\langle\cdot\rangle$ denotes spatial sample averaging. Hence, after multilooking, each pixel in the image is a realization of the $d \times d$ stochastic matrix variable denoted $\mathbf{C}$, and the image is referred to as the multi-look complex covariance image. The dimension $d$ is either 1,2 , or 3 , depending on the scattering vector used. As each channel of the multi-look PolSAR image will be analyzed separately, the one-dimensional case needs to be specified here. For a single channel, i.e. $d=1$, the scattering vector is reduced to a scalar complex scattering coefficient $S$. The data format obtained in multilook domain is

$$
I=\frac{1}{L} \sum_{i=1}^{L} S_{i} S_{i}^{*}
$$

where $I$ is commonly defined as the multilook intensity.

\section{B. Product Model}

In this work, we are concerned about the uniform regions, whose radar cross section may not be perfectly homogeneous, but that are thematically mapped as one class. The well-known product model, described e.g. in [5][13][14], has shown to be mathematically tractable and successful for modeling SAR measurements from single-class regions. In the multilook polarimetric version [13], it decomposes $\mathbf{C}$ into two independent stochastic variables as

$$
\mathbf{C}=T \mathbf{W}
$$

The strictly positive and unit mean scalar random variable $T$ models texture, which is defined here as spatial variation in the mean backscatter due to target variability. In the range of this work, we follow the scalar product model, where the texture variable is regarded as identical in all polarimetric channels. The latter contribution, $\mathbf{W}$, represents the fully developed speckle, which follows the scaled complex Wishart distribution $s \mathcal{W}_{d}(L, \Sigma)[15][16][19]$

$$
f_{\mathbf{W}}(\mathbf{W} ; L, \Sigma)=\frac{L^{L d}}{\Gamma_{d}(L)} \frac{|\mathbf{W}|^{L-d}}{|\boldsymbol{\Sigma}|^{L}} \operatorname{etr}\left(-L \boldsymbol{\Sigma}^{-1} \mathbf{W}\right)
$$

where $\boldsymbol{\Sigma}=E(\mathbf{W})$ is the scale matrix, $|\cdot|$ is the determinant, $\operatorname{etr}(\cdot)=\exp (\operatorname{tr}(\cdot))$ with $\operatorname{tr}(\cdot)$ as the trace operator, $\Gamma_{d}(L)$ is the multivariate gamma function of the complex kind [13], and $L \geq d$ assures that $\mathbf{C}$ is nonsingular. For the homogeneous region characterized by fully developed speckle and no texture, the probability distribution function (PDF) of $T$ is $f_{T}(t)=$ $\delta(t-1)$, where $\delta(\cdot)$ is the Dirac delta function. This results in $\mathbf{C} \sim s \mathcal{W}_{d}(L, \Sigma)$. 
For the general textured region, we need a more complicated distribution for $\mathbf{C}$, which depends on the distribution of $T$. For instance, we obtain the matrix-variate $\mathcal{K}$ distribution [20] for gamma-distributed texture, the $\mathcal{G}_{0}$ distribution [13] for inverse gamma-distributed texture, and the $\mathcal{U}$ distribution [21] for texture that follows a Fisher-Snedecor distribution. The detailed derivations of distributions are given in the mentioned literature.

\section{Mellin-Kind Statistics}

Based on their multiplicative signal model, SAR data have been analyzed on a logarithmic scale for a long time. The log-statistics, also named as Mellin-Kind Statistics (MKS), are used to capture certain characteristics of random variable samples in this domain. The MKS are defined in [17][18][19][22] and evaluated for the distributions of PolSAR data. Here, we repeat the expressions needed in the rest of this paper.

Let $\mathbf{C}$ be a random $d \times d$ matrix defined on the cone $\Omega_{+}$ of positive definite complex Hermitian matrices, whose PDF is $f_{\mathbf{C}}(\mathbf{C})$. The complex Mellin Transform (MT) of a general real-valued function $g(\mathbf{C}): \Omega_{+} \rightarrow \mathbb{R}$ is

$$
G(s)=\mathcal{M}\{g(\mathbf{C})\}(s)=\int_{\Omega_{+}}|\mathbf{C}|^{s-d} g(\mathbf{C}) d \mathbf{C}
$$

with transform variable $s \in \mathbb{C}$. The MT of $f_{\mathbf{C}}(\mathbf{C})$ is defined as the Mellin-kind characteristic function of the random matrix C

$$
\phi_{\mathbf{C}}(s)=E\left\{|\mathbf{C}|^{(s-d)}\right\}=\mathcal{M}\left\{f_{\mathbf{C}}(\mathbf{C})\right\}(s) .
$$

The $\nu$ th-order log-moment is derived from

$$
\mu_{\nu}\{\mathbf{C}\}=E\left\{(\ln |\mathbf{C}|)^{\nu}\right\}=\left.\frac{d^{\nu}}{d s^{\nu}} \phi_{\mathbf{C}}(s)\right|_{s=d}
$$

The Mellin-kind cumulant-generating function is defined as

$$
\varphi_{\mathbf{C}}(s)=\ln \phi_{\mathbf{C}}(s)
$$

and then the $\nu$ th-order log-cumulant is defined as

$$
\kappa_{\nu}\{\mathbf{C}\}=\left.\frac{d^{\nu}}{d s^{\nu}} \varphi_{\mathbf{C}}(s)\right|_{s=d}
$$

As the Mellin-kind characteristic function of a scaled complex Wishart matrix $\mathbf{W}$ with PDF shown in (5) is [22]

$$
\phi_{\mathbf{W}}(s)=\frac{\Gamma_{d}(L+s+d)}{\Gamma_{d}(L)}\left(\frac{|\boldsymbol{\Sigma}|}{L^{d}}\right)^{(s-d)},
$$

the first-order of log-cumulant of fully developed speckle can be derived as:

$$
\kappa_{1}\{\mathbf{W}\}=\ln |\boldsymbol{\Sigma}|+\psi_{d}^{(0)}(L)-d \ln L
$$

where the $\psi_{d}^{(0)}(L)$ is the 0th-order multivariate polygamma function as

$$
\psi_{d}^{(0)}(L)=\sum_{i=0}^{d-1} \psi^{(0)}(L-i),
$$

and $\psi^{(0)}(L)$ is defined as the first logarithmic derivatives of the gamma function

$$
\psi^{(0)}(L)=\frac{d \ln \Gamma(L)}{d L}
$$

Based on the multilook polarimetric product model, the Mellin-kind characteristic function is expressed as [22]

$$
\phi_{\mathbf{C}}(s)=\phi_{T}(d(s-d)+1) \phi_{\mathbf{W}}(s)
$$

where $\phi_{T}(s)$ is the univariate Mellin-kind characteristic function of a general texture random variable $T$. This yields the population log-cumulants [22]

$$
\kappa_{\nu}\{\mathbf{C}\}=\kappa_{\nu}\{\mathbf{W}\}+d^{\nu} \kappa_{\nu}\{T\}
$$

Thus, the first-order log-cumulant of $\mathbf{C}$ evaluate under the product model to

$$
\kappa_{1}\{\mathbf{C}\}=\ln |\boldsymbol{\Sigma}|+\left(\psi_{d}^{(0)}(L)-d \ln L\right)+d \kappa_{1}\{T\}
$$

In addition, based on the relation between the log-cumulant and log-moment of $\mathbf{C}$ [22],

$$
\kappa_{\nu}\{\mathbf{C}\}=\mu_{\nu}\{\mathbf{C}\}-\sum_{i=1}^{\nu-1}\left(\begin{array}{c}
\nu-1 \\
i-1
\end{array}\right) \kappa_{i}\{\mathbf{C}\} \mu_{\nu-i}\{\mathbf{C}\},
$$

the first-order log-cumulant follows as

$$
\kappa_{1}\{\mathbf{C}\}=\mu_{1}\{\mathbf{C}\}=E\{(\ln |\mathbf{C}|)\},
$$

Therefore, the expressions leads to

$$
E\{(\ln |\mathbf{C}|)\}=\ln |E\{\mathbf{C}\}|+\left(\psi_{d}^{(0)}(L)-d \ln L\right)+d \kappa_{1}\{T\} .
$$

The above property in eq. (20) also applies to one-dimension case, i.e. $d=1$, just replacing the matrix $\mathbf{C}$ with the scalar multilooking intensity $I$ as follows,

$$
E\{\ln I\}=\ln E\{I\}+\left(\psi^{(0)}(L)-d \ln L\right)+\kappa_{1}\{T\} .
$$

The mentioned ML estimator for ENL is the maximum likelihood solution of parameter $L$ in the eq. (12), the first-order log-cumulant equation of the complex Wishart distribution. In our former work [11], we use the one-dimensional version of the ML estimator to obtain ENL in each polarimetric channel. As we found, in the regions containing mixture of multiple classes, the estimator will produce strongly deviating ENL values in different polarimetric channels. It indicates that the inconsistency of the first-order log-cumulant between polarimetric channels may reflect the presence of mixture between classes. Based on this idea, we start the investigation of a test statistic in the next section.

\section{Methodology}

We take $U$ as the set of uniform windows. Then the presence of non-uniformity in window $w_{i}$ is determined by choosing between the hypotheses:

$$
\begin{aligned}
& H_{0}: w_{i} \in U \\
& H_{1}: w_{i} \notin U .
\end{aligned}
$$

The null hypothesis $H_{0}$ corresponds to uniformity and the alternative hypothesis $H_{1}$ to non-uniformity.

\section{A. Derivation of Test Statistic}

Firstly we investigate a test statistic for the hypothesis test. 
1) Uniform windows: For a uniform region where the pixels are realization of the same stochastic process, the firstorder log-cumulants of multilook intensities of two different polarimetric channels, denoted $a$ and $b$, can be expressed by the equations:

$$
\begin{aligned}
& E\left\{\ln I_{a}\right\}=\ln E\left\{I_{a}\right\}+D(L)+\kappa_{1}(T) . \\
& E\left\{\ln I_{b}\right\}=\ln E\left\{I_{b}\right\}+D(L)+\kappa_{1}(T) .
\end{aligned}
$$

Here $I_{a}$ and $I_{b}$ represent the intensities in the two polarimetric channels, and $D(L)=\left(\psi^{(0)}(L)-\ln L\right)$ is a constant. In the scalar texture product model, $\kappa_{1}(T)$ is identical for all polarimetric channels. Therefore, it follows that

$$
\left[\ln E\left\{I_{a}\right\}-E\left\{\ln I_{a}\right\}\right]-\left[\ln E\left\{I_{b}\right\}-E\left\{\ln I_{b}\right\}\right]=0 .
$$

2) Non-uniform windows: We assume the analyzed nonuniform regions only to contain mixture of two classes. This simplification is reasonable, as the windows used for small sample ENL estimates are usually in a size of $3 \times 3$ or $5 \times 5$ [9][10] and the probability that they will contain more than two classes is low. Suppose a region contains two classes, class 1 and class 2 . Let $I_{a}^{(1)}$ represent the intensity of class 1 in polarimetric channel $a$, for example. Then the intensity ratio between class 1 and 2 in channel $a$ can be denoted by $R_{m}=$ $E\left\{I_{a}^{(1)}\right\} / E\left\{I_{a}^{(2)}\right\}$. The prior probabilities of these two classes within the region are assumed as $p_{1}$ and $p_{2}$, respectively, such that $p_{1}+p_{2}=1$. We can deduce that

$$
\begin{aligned}
& {\left[\ln E\left\{I_{a}\right\}-E\left\{\ln I_{a}\right\}\right]-\left[\ln E\left\{I_{b}\right\}-E\left\{\ln I_{b}\right\}\right]} \\
& =\ln \frac{p_{1} R_{a}^{1-p_{1}}+\left(1-p_{1}\right) R_{a}^{-p_{1}}}{p_{1} R_{b}^{1-p_{1}}+\left(1-p_{1}\right) R_{b}^{-p_{1}}}
\end{aligned}
$$

It can be verified that only when $R_{a}$ and $R_{b}$ happen to be two distinct roots of the equation

$$
p_{1} r^{1-p_{1}}+\left(1-p_{1}\right) r^{-p_{1}}=\text { Const }
$$

where $r$ is the unknown in the equation and Const is a constant larger than 1, then eq. (26) could hold for a non-uniform window. Such a special case exists in a mathematical sense, but in most real data it will not appear. That is, for non-uniform windows, $\left[\ln E\left\{I_{a}\right\}-E\left\{\ln I_{a}\right\}\right]-\left[\ln E\left\{I_{b}\right\}-E\left\{\ln I_{b}\right\}\right]$ will in practice be unequal to 0 . All the details of the proof are shown in the appendix A.

Based on the above analysis, for the uniform region the statistic $\ln E\{I\}-E\{\ln I\}$ should be equal in every polarimetric channel. The statistic $X_{a}$ is defined as

$$
X_{a}=\ln \left\langle I_{a}>-<\ln I_{a}>, a \in\{H H, \text { Cross, } V V\}\right.
$$

where the subscripts $a$ represents the channels of the PolSAR data, amd $\langle\cdot\rangle$ represents the sample mean. Then an oneway ANOVA can be adopted first to determine whether the $X_{H H}, X_{C r o s s}$ and $X_{V V}$ samples are all from the populations with equal mean. If the dataset passes the one-way ANOVA, then it means the whole image is uniform and can be directly used for ENL estimation. Otherwise, we need to test every window. The difference of $X_{a}$ can be taken as test statistic, which is formulated as

$$
\begin{gathered}
\Delta X_{a b}=\left[\ln <I_{a}>-<\ln I_{a}>\right]-\left[\ln <I_{b}>-<\ln I_{b}>\right], \\
a, b \in\{H H, \text { Cross }, V V\}, a \neq b
\end{gathered}
$$

The possible test statistics can be separated into three pairs based on the polarimetric channels involved. For example, $\Delta X_{H H, V V}$ and $\Delta X_{V V, H H}$ are taken as a pair, since both of them are determined by data in the $\mathrm{HH}$ and $\mathrm{VV}$ polarimetric channels.

\section{B. Retrieval of Sampling Distribution}

We calculate the test statistics in sliding windows over an arbitrary image. The realization of $\Delta X_{a b}$ in a certain window $w_{i}$ is defined as $\Delta X_{a b}^{w_{i}}$. The overall sampling distribution, denoted as $f\left(\Delta X_{a b}^{w_{i}}\right)$, can be estimated from the collection of values calculated over the whole image. This empirical distribution will depend strongly upon the properties of the given image, i.e., the extent of the uniform regions. Due to the unpredictable shape and possible multimodality of the distribution, we propose to estimate it with a kernel density estimator (KDE) implemented with the Epanechnikov kernel function [23][24].

The sampling distribution of $\Delta X_{a b}$ from uniform windows and non-uniform windows can be expressed as $f\left(\Delta X_{a b}^{w_{i}} \mid w_{i} \in\right.$ $U)$ and $f\left(\Delta X_{a b}^{w_{i}} \mid w_{i} \notin U\right)$, respectively. It is difficult to derive an exact expression for the sampling distribution $f\left(\Delta X_{a b}^{w_{i}} \mid w_{i} \in U\right)$, which is required for uniform window detection. Here we introduce a general approach to retrieve the approximate $f\left(\Delta X_{a b}^{w_{i}} \mid w_{i} \in U\right)$ from the overall sampling distribution $f\left(\Delta X_{a b}\right)$ of an arbitrary image.

As the overall distribution is contributed by values from both uniform and non-uniform windows, it can be formulated as:

$f\left(\Delta X_{a b}^{w_{i}}\right)=\pi_{u} \cdot f\left(\Delta X_{a b}^{w_{i}} \mid w_{i} \in U\right)+\pi_{n u} \cdot f\left(\Delta X_{a b}^{w_{i}} \mid w_{i} \notin U\right)$.

where the $\pi_{u}$ and $\pi_{n u}$ represent the prior probabilities of uniform and non-uniform windows, respectively, over a particular image, and $\pi_{u}+\pi_{n u}=1$. As derived above, the mathematical expectation of $\Delta X_{a b}$ is zero for the uniform windows. The corresponding sampling distribution $f\left(\Delta X_{a b}^{w_{i}} \mid w_{i} \in U\right)$ should be symmetric and zero-mean. It satisfies

$$
f\left(\Delta X_{a b}^{w_{i}} \mid w_{i} \in U\right)=f\left(-\Delta X_{a b}^{w_{i}} \mid w_{i} \in U\right) .
$$

In addition, the paired test statistics, $\Delta X_{a b}$ and $\Delta X_{b a}$, are the opposite to each other. For any window $w_{i}$, it means that

$$
\Delta X_{a b}^{w_{i}}=-\Delta X_{b a}^{w_{i}} .
$$

Therefore, we have

$$
f\left(\Delta X_{a b}^{w_{i}} \mid w_{i} \in U\right)=f\left(\Delta X_{b a}^{w_{i}} \mid w_{i} \in U\right),
$$

stating that the zero-mean symmetric distributions of sample values from the uniform windows should be identical for the paired test statistics. The samples from non-uniform windows have in general distributions with non-zero mean. Thus $f\left(\Delta X_{a b}^{w_{i}} \mid w_{i} \notin U\right)$ is mainly distributed to one side of the zero value, while $f\left(\Delta X_{b a}^{w_{i}} \mid w_{i} \notin U\right)$ is distributed mainly to the other side. It leads the two overall distributions to present positive and negative skewness.

As shown in the schematic diagram of Fig. 1, if we stack up the distributions of $\Delta X_{a b}$ (red) and $\Delta X_{b a}$ (blue), 


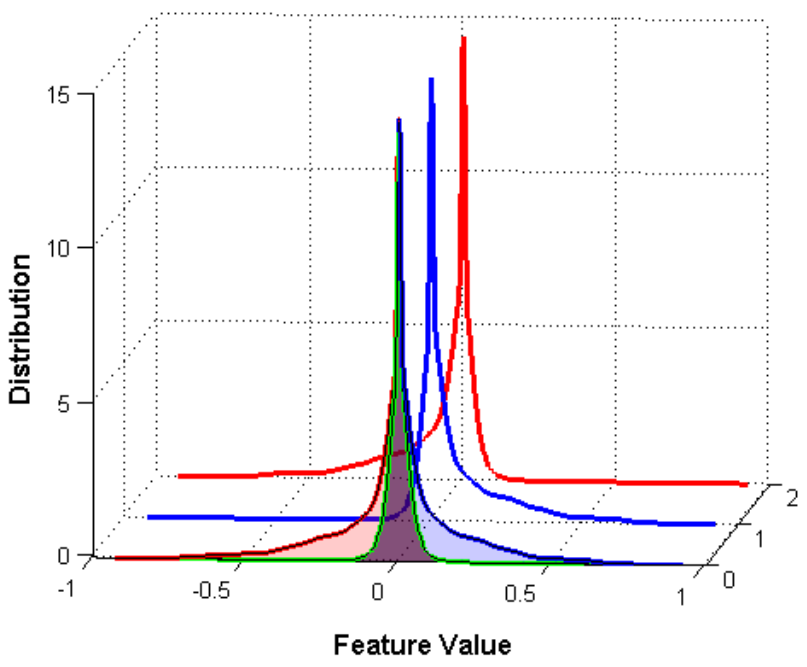

Fig. 1: Schematic diagram which explains how the sampling distribution for uniform windows is approximated. The distributions in red and blue represent the paired test statistics $\Delta X_{a b}$ and $\Delta X_{b a}$, respectively. Their overlapping part is outlined in green.

the overlapping part outlined in green is the minimum of $f\left(\Delta X_{a b}^{w_{i}}\right)$ and $f\left(\Delta X_{b a}^{w_{i}}\right)$. It follows that

$$
\begin{aligned}
& \quad \min \left\{f\left(\Delta X_{a b}^{w_{i}}\right), f\left(\Delta X_{b a}^{w_{i}}\right)\right\} \\
& =\min \left\{\pi_{u} f\left(\Delta X_{a b}^{w_{i}} \mid w_{i} \in U\right)+\left(1-\pi_{u}\right) f\left(\Delta X_{a b}^{w_{i}} \mid w_{i} \notin U\right),\right. \\
& \left.\quad \pi_{u} f\left(\Delta X_{b a}^{w_{i}} \mid w_{i} \in U\right)+\left(1-\pi_{u}\right) f\left(\Delta X_{b a}^{w_{i}} \mid w_{i} \notin U\right)\right\} \\
& =\pi_{u} f\left(\Delta X_{a b}^{w_{i}} \mid w_{i} \in U\right) \\
& \quad+\left(1-\pi_{u}\right) \min \left\{f\left(\Delta X_{a b}^{w_{i}} \mid w_{i} \notin U\right), f\left(\Delta X_{b a}^{w_{i}} \mid w_{i} \notin U\right)\right\},
\end{aligned}
$$

where the $\min \{\cdot, \cdot\}$ represents the function that takes the minimum values between the two inputs. Comparing eq. (31) and eq. (35), the component contributed by non-uniform windows is significantly reduced in the $\min \left\{f\left(\Delta X_{a b}^{w_{i}}\right), f\left(\Delta X_{b a}^{w_{i}}\right)\right\}$ as the overlapping part of $f\left(\Delta X_{a b}^{w_{i}} \mid w_{i} \notin U\right)$ and $f\left(\Delta X_{b a}^{w_{i}} \mid w_{i} \notin\right.$ $U)$ is relatively small. Therefore $\min \left\{f\left(\Delta X_{a b}^{w_{i}}\right), f\left(\Delta X_{b a}^{w_{i}}\right)\right\}$ after rescaled by $1 / \pi_{u}$ can be used to describe $f\left(\Delta X_{a b}^{w_{i}} \mid w_{i} \in\right.$ $U$ ) approximately, which is denoted as

$$
\hat{f}\left(\Delta X_{a b}^{w_{i}} \mid w_{i} \in U\right)=1 / \pi_{u} \cdot \min \left\{f\left(\Delta X_{a b}^{w_{i}}\right), f\left(\Delta X_{b a}^{w_{i}}\right)\right\} .
$$

However, for an arbitrary image, the prior probability $\pi_{u}$ for uniform windows is unknown. Then in practice, we rescale the $\min \left\{f\left(\Delta X_{a b}^{w_{i}}\right), f\left(\Delta X_{b a}^{w_{i}}\right)\right\}$ by making the approximated distribution $\hat{f}\left(\Delta X_{a b}^{w_{i}} \mid w_{i} \in U\right)$ integrate to one.

\section{Threshold Selection}

The two hypotheses in (22) and (23) can be quantitatively distinguished by selecting thresholds based on the proposed test statistic $\Delta X_{a b}$. We use the approximated sampling distribution for uniform windows, $\hat{f}\left(\Delta X_{a b}^{w_{i}} \mid w_{i} \in U\right)$, to represent $H_{0}$, denoted as $f\left(\Delta X_{a b}^{w_{i}} \mid H_{0}\right)$. As the $H_{0}$ distribution is symmetrically distributed around 0, a two-sided test should be given by

$$
T_{l o} \underset{H_{0}}{\stackrel{H_{1}}{>}} \Delta X_{a b}^{w_{i}} \stackrel{H_{1}}{\underset{H_{0}}{>}} T_{u p}
$$

where the inequalities state the hypothesis decisions. For a desired significance level $\alpha$, the thresholds $T_{u p}$ and $T_{l o}$ define the upper and lower $\alpha / 2$ quantiles of $f\left(\Delta X_{a b}^{w_{i}} \mid H_{0}\right)$, respectively. Based on the Bonferroni correction, the significance level adopted for each pairwise test here should equal to $1 / 3$ of the desired value. As the distributions are exactly symmetric about zero, $T_{l o}$ is actually the negative of $T_{u p}$, that is, $T_{l o}=-T_{u p}$. We can obtain required thresholds $T_{u p}$ by solving

$\alpha=\int_{-\infty}^{-T_{u p}} f\left(t \mid H_{0}\right) d t+\int_{T_{u p}}^{\infty} f\left(t \mid H_{0}\right) d t=2 \int_{T_{u p}}^{\infty} f\left(t \mid H_{0}\right) d t$

We also propose another criteria to determine the thresholds by limiting the proportion of the non-uniformity windows in the whole estimation windows accepted by $H_{0}$. The overall distribution of a certain test statistic can be divided into two parts. One part, which is the overlap between distributions of the two paired test statistics, $\min \left\{f\left(\Delta X_{a b}^{w_{i}}\right), f\left(\Delta X_{b a}^{w_{i}}\right)\right\}$, represents the samples from uniform windows, while the other part represents the samples from non-uniform windows. Then the non-uniformity ratio $R_{n u}$ is expressed as the percentage of non-overlap area within the whole area of the overall distribution between thresholds $\pm T_{u p}$, as follows:

$$
R_{n u}=1-\frac{\int_{-T_{u p}}^{T_{u p}} \min \left\{f\left(\Delta X_{a b}^{w_{i}}\right), f\left(\Delta X_{b a}^{w_{i}}\right)\right\} d t}{\int_{-T_{u p}}^{T_{u p}} f\left(\Delta X_{a b}^{w_{i}}\right) d t},
$$

where $t$ represents the continuous variable in sample space. Therefore, the threshold can be retrieved from eq. (39) for the given $R_{n u}$, which has more explicit physical meaning than significance level. In the experiments, we set the $R_{n u}=10 \%$.

Then the hypothesis test is applied to test statistics from all windows. If the $H_{0}$ hypothesis is accepted for each $\Delta X_{a b}$ from a particular window, then this window can be regarded as uniform. Otherwise it is taken as non-uniform and is not used towards the final ENL estimate.

A simplification which should be pointed out is that not every test statistic needs to be hypothesis tested. According to eq. (33), for any sample $\Delta X_{a b}^{w_{i}}$ which satisfies the $-T_{u p}<\Delta X_{a b}^{w_{i}}<T_{u p}$, its paired value from the same window, $\Delta X_{b a}^{w_{i}}$, will also fall between the desired thresholds. It means that the test results are exact the same for both test statistics in a pair. Therefore, we only need to apply the hypothesis test on one of the paired statistics. In following experiments based on three-dimensional PolSAR data, the hypothesis test is just implemented on the three test statistics, $\Delta X_{H H, V V}, \Delta X_{H H, C r o s s}$ and $\Delta X_{C r o s s, V V}$. As the windows containing mixture of different classes are removed through the hypothesis test, we define it as the mixture-eliminating (ME) procedure.

\section{Complete Estimation Process}

After the ME procedure, we apply the full-polarimetric ENL estimators, like ML and DTM, to the covariance matrices 
within each accepted window. Then the small sample ENL estimates are collected, producing a one-dimensional empirical distribution. Now the assumption of dominantly uniform windows is much more likely to be satisfied. Then we can take the mode value as the global ENL estimate with a greater degree of confidence.

The ML and DTM estimators are detailed in [9] and [10], respectively. The ML estimator is based on a statistical model for fully developed speckle. It has a superior performance and achieves low bias and variance ENL estimates for Wishart distributed regions, but it will produce underestimated results for heterogeneous regions that contain texture or mixtures of different classes. The DTM estimator is claimed to be textureinvariant based on the scalar product model, but its variance is relatively high. We name the traditional unsupervised smallsample ENL estimation methods directly after their adopted estimators as ML or DTM. The modified methods, where the windows are prescreened by the proposed ME procedure, are abbreviated as MEML or MEDTM.

In summary, the general block diagram of our unsupervised mixture-eliminating ENL estimator for multipolarization SAR data is shown in Fig. 2, which is made up of 8 main steps.

1. Calculate the statistic $X_{a}$ of each polarimetric channel in sliding windows over the PolSAR image.

2. Implement the one-way ANOVA on the combination of samples. If the samples pass the ANOVA test, then the smallsample ENL estimation can be directly applied on all the windows. Otherwise the process goes into the step 3.

3. Take the difference of the statistic between channels, $\Delta X_{a b}$, as test statistic. With different channel permutations, three pairs of $\Delta X_{a b}$ will be achieved.

4. Stack up the two distributions of each paired test statistics and use the rescaled overlapping part to approximate the sampling distribution of the test statistic for uniform windows.

5. Compute the thresholds based on the desired significance level or non-uniformity ratio $R_{n u}$ for each test statistic pair.

6. Implement the hypothesis test on the collections of test statistic, $\Delta X_{H H, V V}, \Delta X_{H H, C r o s s}$ and $\Delta X_{C r o s s, V V}$ in our cases, with corresponding selected thresholds. Keep the windows for which the $H_{0}$ hypothesis is accepted by all tests for further ENL estimation.

7. Apply the full-polarimetric ENL estimator to the remaining windows and produce an empirical distribution of small sample ENL estimates.

8. Take the mode values as the global ENL estimate.

\section{EXPERIMENT}

In this section, we first use simulated data to show the capability of the proposed approach to distinguish uniform and non-uniform windows, and then use real data to compare the performance of the small-sample ENL estimation methods with and without the mixture-eliminating procedure.

\section{A. Simulated Data}

In the first experiment, we generate a four-class test PolSAR image to validate the uniform window detector. The synthetic data set consists of $120 \times 120$ three-dimensional

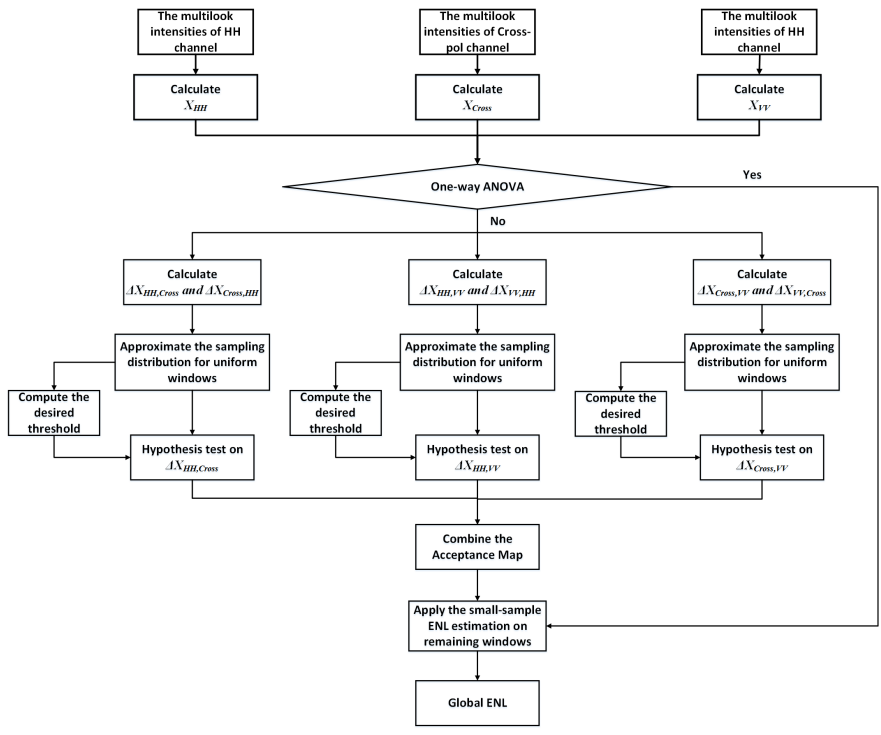

Fig. 2: The general block diagram of the proposed unsupervised mixture-eliminating ENL estimation algorithm for PolSAR data

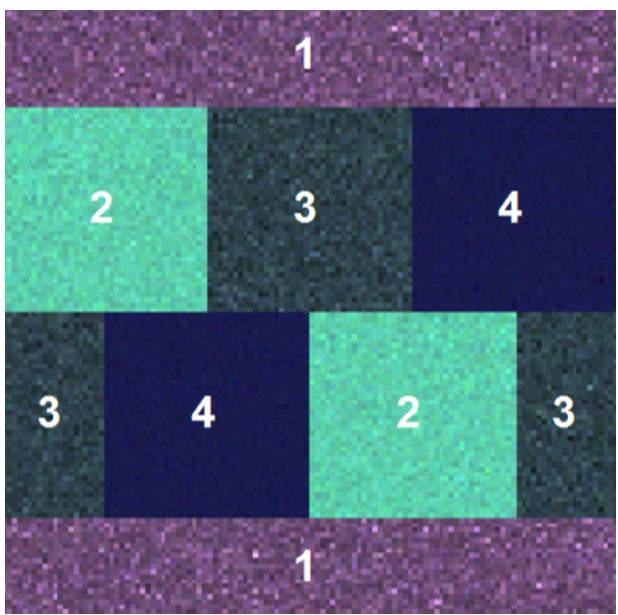

Fig. 3: Pauli decomposition composite image with class labels. The shown simulated data are multi-looked by 25 .

covariance matrix samples in total. The samples from each class are drawn from a $\mathcal{U}$ distribution [21]. The four classes are simulated with the polarimetric properties of a dense urban area, a rotated building area, a vegetated mountain area and water, respectively. To be realistic, the class-specific texture parameters and mean covariance matrices are retrieved from four corresponding uniform regions in real images. The Pauli decomposition composite image of the simulated pattern is shown in Fig. 3 with class labels.

1) Approximated Sampling Distribution: Fig. 4 shows a label map based on ground truth of the test pattern. The sliding windows that contain only one class are labeled as uniform and the transboundary windows are labeled as non-uniform. We calculate the three pairs of test statistics in every window across the data set. Taking the collections of $\Delta X_{H H, V V}$ values for example, its overall distribution is displayed in Fig. 5. Here, we use blue color to represent the contribution of the 


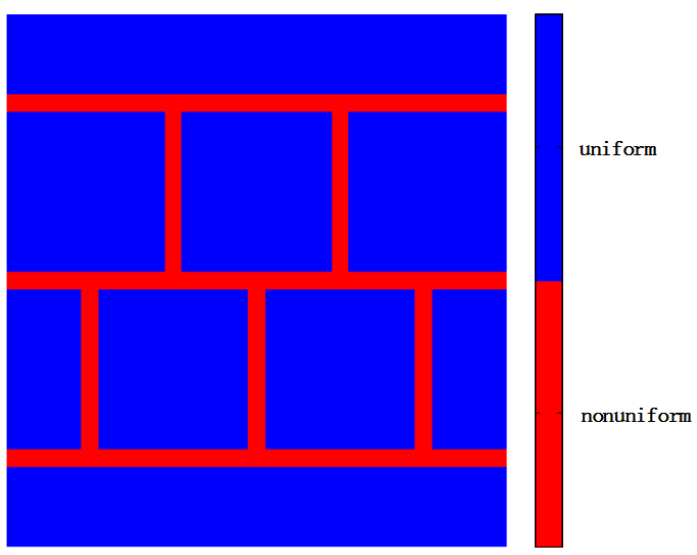

Fig. 4: Predefined label map based on ground truth.

values from the predefined uniform windows and red for the remaining samples. The overall distribution is outlined in black. For this simulated data set, the distribution of $\Delta X_{H H, V V}$ is of positive skewness, therefore its paired statistic $\Delta X_{V V, H H}$ should have a negative skewed distribution. The distributions of $\Delta X_{H H, V V}$ and $\Delta X_{V V, H H}$ are stacked up as shown in the top panel of Fig. 6, and the overlapping part is outlined in green. The same process is conducted on the other two pairs. The result for $\Delta X_{H H, C r o s s}$ and $\Delta X_{C r o s s, H H}$ is demonstrated in the middle panel of Fig. 6, and that for $\Delta X_{V V, C r o s s}$ and $\Delta X_{C r o s s, V V}$ is displayed in the bottom panel of Fig. 6. In all cases shown in Fig. 6, the majority of the overlap regions are occupied by the samples from uniform windows. Besides, to quantify the approximation, we calculated the Kolmogorov distance between the empirical distribution and the approximated one. The results are shown in Table. I. Note that the distance is rather small (from 0.0081 to 0.0138 ) in all cases. It indicates the feasibility to approximate the sampling distribution for uniform windows by the corresponding overlap.

TABLE I: Kolmogorov distance between the empirical distribution of different test statistic and the approximated distribution.

\begin{tabular}{lccc}
\hline Test Statistic & $\Delta X_{H H, V V}$ & $\Delta X_{H H, \text { Cross }}$ & $\Delta X_{\text {Cross }, V V}$ \\
\hline Kolmogorov distance & 0.0138 & 0.0081 & 0.0081 \\
\hline
\end{tabular}

2) Evaluation of Threshold: The fore-mentioned nonuniformity ratio $R_{n u}$ is adopted to determine the threshold for the following hypothesis tests. Based on the simulated data sets, the changes of $R_{n u}$ values with the threshold on the three pairs of test statistics are shown in Fig. 7. The $R_{n u}$ increases in general with the selected threshold. The $R_{n u}$ rises quickly when the threshold is relatively low, and then the growth slows down and stabilizes gradually. In the experiment, the $R_{n u}$ is set to be $10 \%$, where its sensitivity to the threshold is moderate. From the changing curve, we can obtain the threshold for $\Delta X_{H H, V V}, \Delta X_{H H, \text { Cross }}$ and $\Delta X_{C r o s s, V V}$, as $0.55,0.92$ and 1.05 , respectively. Then the hypothesis test is implemented on the sample collections of the three test statistics. The final acceptance map is shown in
Fig. 8, where the blue parts represent the windows detected as uniform while the red parts are the non-uniform ones. As displayed, most windows crossing class boundaries are taken as non-uniform, as we expected, and within the highly textured classes, like class 1 , the probability of a uniform window to be taken as non-uniform also increases.

We compare the resulting uniformity map with one other conventional homogeneity metric, the standard coefficient of variation $(\mathrm{CV})$. The calculation of $\mathrm{CV}$ is usually based on the single channel data. For the full-polarimetric SAR data, the sample matrices can be projected to one-dimension by calculating corresponding SPAN value[12], which is defined as

$$
S P A N=I_{H H}^{2}+I_{C r o s s}^{2}+I_{V V}^{2}
$$

Then the CV can be obtained with SPAN data as

$$
C V=\frac{\operatorname{std}(S P A N)}{\operatorname{mean}(S P A N)}
$$

where the $\operatorname{std}(\cdot)$ and mean $(\cdot)$ represent the calculation of the sample standard variation and sample mean, respectively. When evaluating the homogeneity with CV metric, usually the regions with relative low $\mathrm{CV}$ value are taken as homogeneous while the regions with high $\mathrm{CV}$ value are regarded as heterogeneous. To compare the performance of CV-based method and our proposed strategy in distinguishing the uniform and non-uniform windows, we can obtain the uniformity maps of both methods under the same separation ratio and then visually determine which is closer to the standard uniformity map shown in Fig. 4. In the uniformity map obtained by proposed method, the percentage of non-uniform windows is about 14\%. Therefore with CV-based method, the windows with $14 \%$ largest CV values are taken as non-uniform windows. Then the corresponding uniformity map of this method can be obtained and shown in Fig. 9. It is noticeable that under $\mathrm{CV}$ metric, some boundary areas fail to be regarded as nonuniform windows while massive windows in the regions of class 1 are taken as class mixture. Comparing the Fig. 8 and Fig. 9, the uniformity map obtained by proposed method is obviously closer to the standard map in Fig. 4.

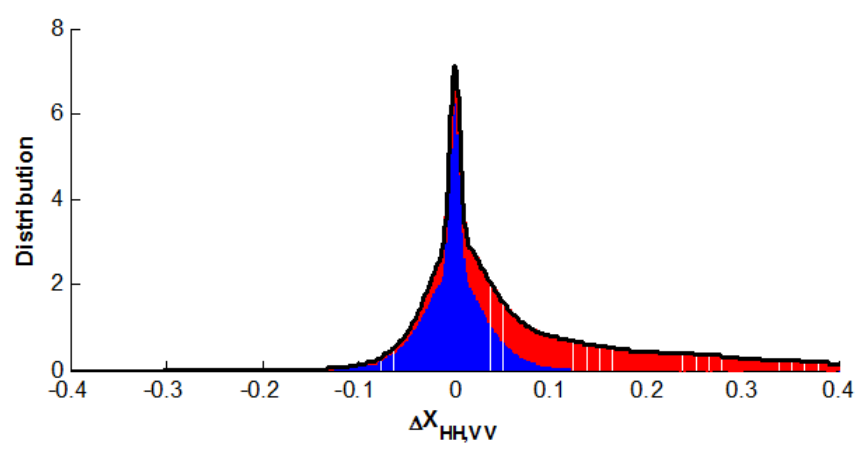

Fig. 5: Overall Distribution (outlined in black) of $\Delta X_{H H, V V}$ over the simulated images: the combination of the values from the predefined uniform windows (blue) and non-uniform windows (red) 

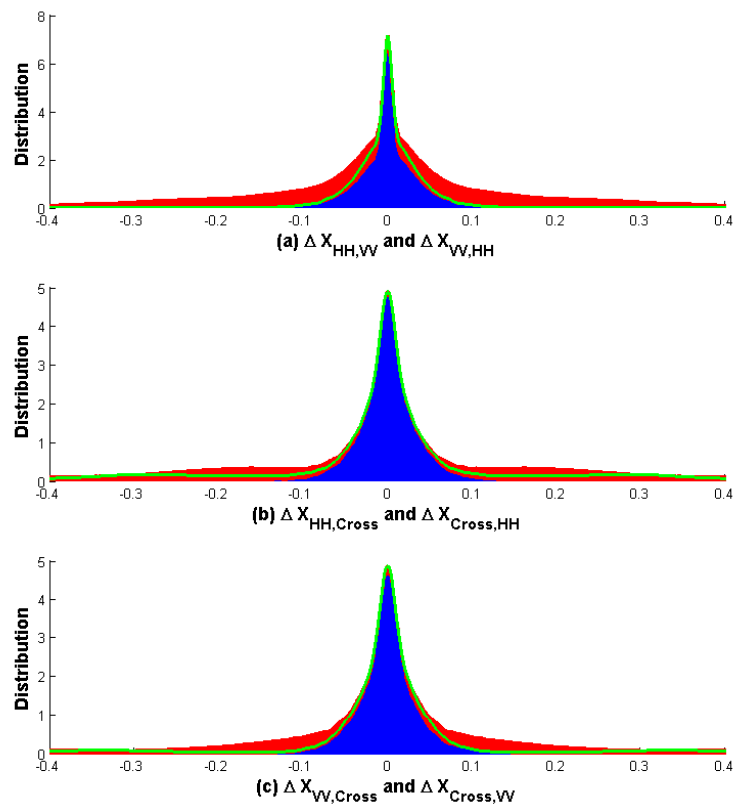

Fig. 6: The overlapped regions (outlined in green) achieved by stacking up the two overall distributions of test statistics in a pair: (a) $\Delta X_{H H, V V}$ and $\Delta X_{V V, H H}$, (b) $\Delta X_{H H, C r o s s}$ and $\Delta X_{C r o s s, H H}$, (c) $\Delta X_{V V, C r o s s}$ and $\Delta X_{C r o s s, V V}$. The blue and red parts correspond to the contribution of uniform and non-uniform windows as shown in Fig. 5

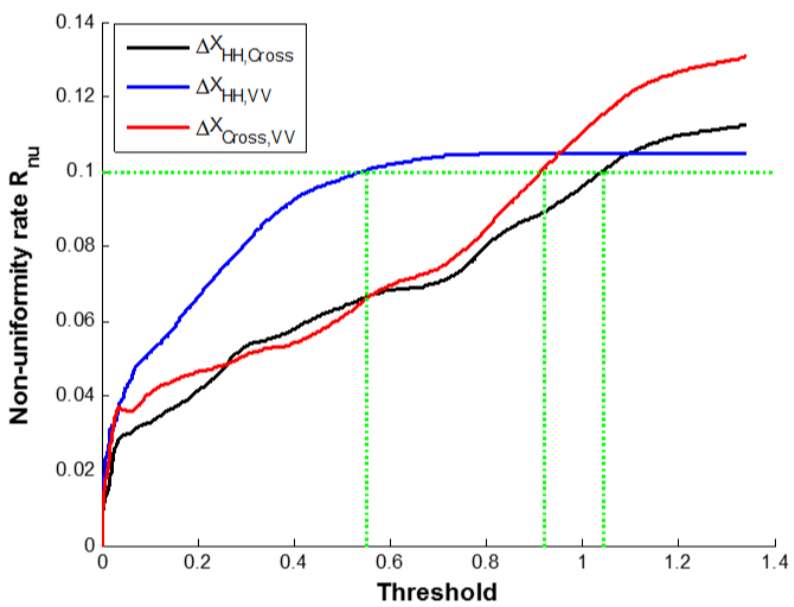

Fig. 7: The changing curve of non-uniformity ratio with the threshold value.

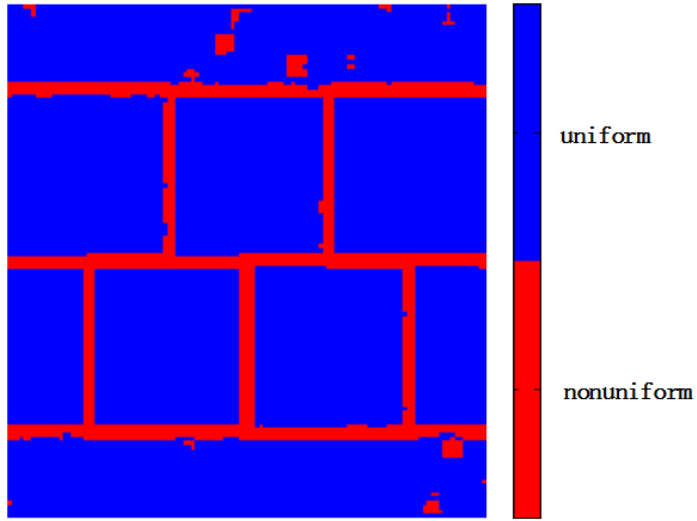

Fig. 8: The final acceptance map.

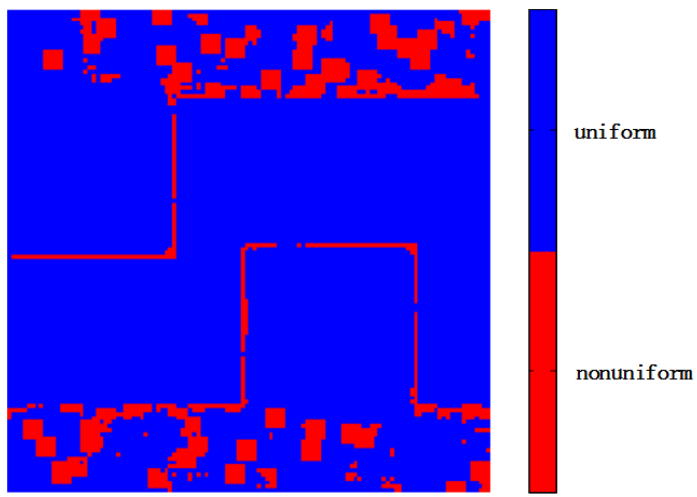

Fig. 9: Uniformity map obtained by CV metric.

3) Effect of Nominal Number of Looks: For various application, different degree of multi-looking will be implemented. It is necessary to investigate the effect of preset looks on the uniform window detector, in order to assess the potency of the proposed strategy for different applications. The simulated data set is multi-looked by $1,4,9,16,25$ and 36, respectively. We use the precision rate and corresponding false alarm rate to evaluate the performance of the uniform window detector. Here, the precision rate $P_{d}$ refers to the rate of actual uniform windows in all passing the hypothesis tests, which is formulated as

$$
P_{d}=\frac{\operatorname{card}\left(\left\{w_{i} \mid w_{i} \in U, \Delta X_{a b}^{w_{i}} \in\left[-T_{u p}, T_{u p}\right]\right\}\right)}{\operatorname{card}\left(\left\{w_{i} \mid \Delta X_{a b}^{w_{i}} \in\left[-T_{u p}, T_{u p}\right]\right\}\right)}
$$

where $\operatorname{card}(\cdot)$ represents the function to calculate the number of elements in the input set.

Considering the randomness in the simulated data, we carry out 10 Monte Carlo experiments for each preset number of looks. The changing curves of averaged precision rate and false alarm rate are shown in Fig. 10. We notice that the precision rate increases with the nominal number of looks. The growth between the case of single look data and 4-look data is most significant, while the changing between the other cases is relative slow. This is because that the Mellin statistic of the single data, which does not follow $L \leq d$, can not be formulated by the (21). Thus the proposed uniform window detector is not exactly suitable for single look data. But as shown in Fig. 10, even for the single-look dataset, the precision 


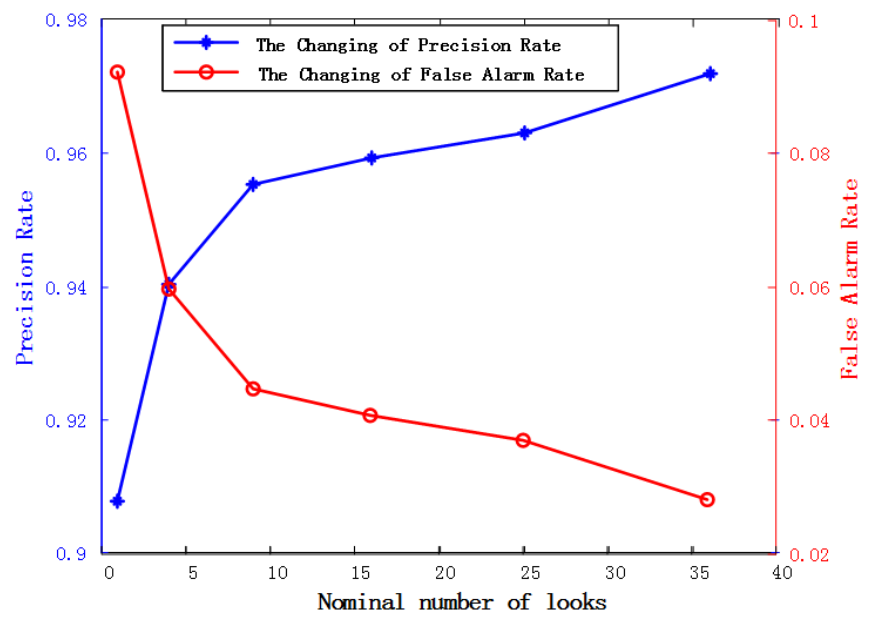

Fig. 10: The changing of averaged precision rate and false alarm rate with nominal number of looks.

rate has reached almost $91 \%$. Therefore, the detector is capable to perform on data under different number of looks in general.

4) Effect of Window Size: Based on the precision rate, we can study the evolution of the detector's performance as window size increases from $3 \times 3$ to $9 \times 9$. The uniformity maps obtained in all cases are shown in Fig. 11. Comparing these maps, with larger size more windows located close to class boundary are labeled as non-uniform. It accords with the cognition that the number of windows containing mixture of classes increases with sliding window size. Based on the ground truth of the simulated image, the precision rate for each case can be achieved, which is shown in Fig. 12. The resulting precision rates vary between cases, but all remain at high level. It means for different window size, the most detected uniform windows do contain single class and can be adopted for further ENL estimation phase. Since the window size do not have significant effect on the proposed detector, it should choose the optimal size for the small-sample estimation, which has been discussed in [9]. Therefore, based on Anfinsen's investigation, we adopt window with $5 \times 5$ in size for the following experiments.

\section{B. Real Data}

After showing the feasibility to distinguish the uniform and non-uniform windows with synthetic data, we turn to real data for a realistic comparison between the performance of the unsupervised ENL estimation approaches before and after incorporating the mixture-eliminating procedure into the whole process. Two polarimetric SAR images are used in the experiments. The chosen data sets are both acquired by the Radarsat-2 C-band instrument: one is an image of a cross-sea bridge in Flevoland, Netherlands, and one is an image of the San Francisco Bay area in California, USA. Both data sets contain covariance matrices with nominal number of looks equal to 25. The Pauli decomposition composite images of these two data sets are shown in Fig. 13 and 14.

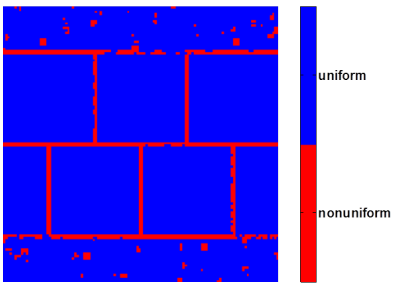

(a) $3 \times 3$

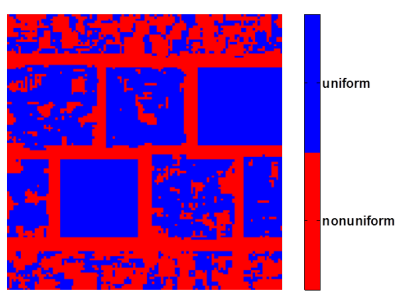

(c) $7 \times 7$

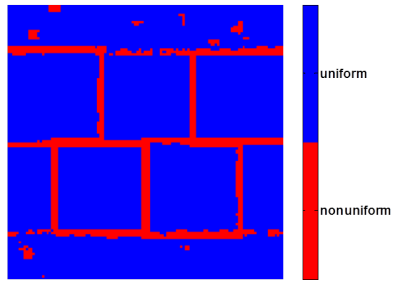

(b) $5 \times 5$

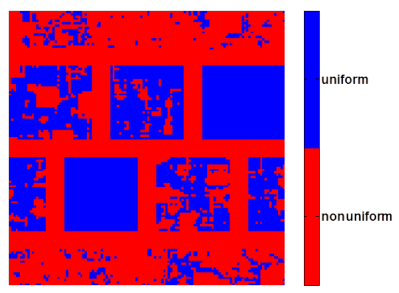

(d) $9 \times 9$
Fig. 11: The obtained uniformity map under different window size

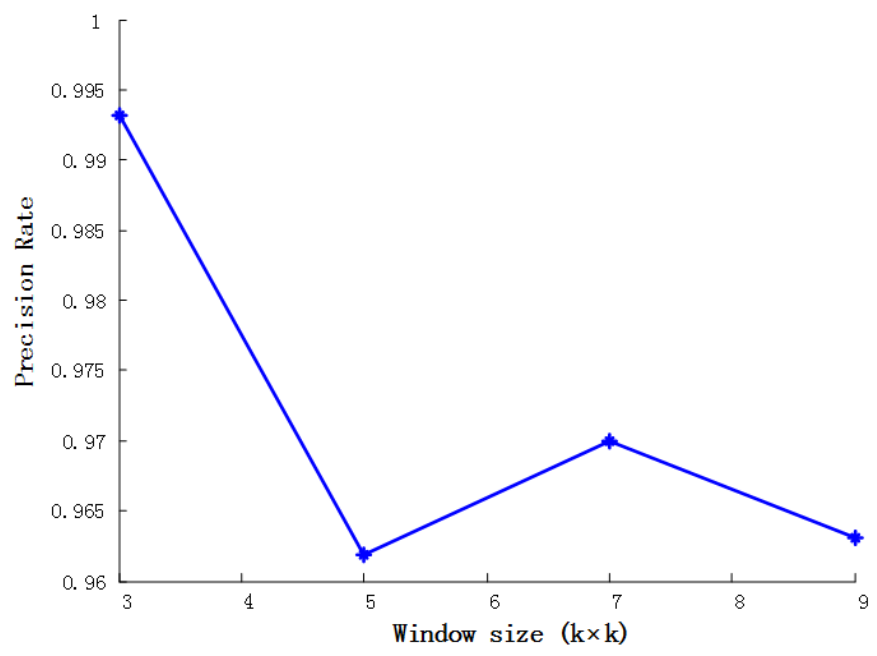

Fig. 12: The precision rate under different window size

The two images are used to demonstrate the robustness of the unsupervised ENL estimators to different complexity of the SAR scenes. The first image consists mainly of a homogeneous ocean area and a bridge crossing it. The San Francisco Bay image contains mostly sea and urban areas, as well as some parks and hills covered by vegetation. There are few homogeneous areas of considerable size, except for the ocean. Obviously the San Francisco image is a more complicate scene and therefore provides less uniform windows for ENL estimation.

Each image was processed with a sliding estimation window of size $5 \times 5$ pixels to cover the whole image. No speckle filter was applied initially. For the original small-sample estimation approaches, the ENL estimator, ML or DTM, is directly applied to every window. In the modified MEML and MEDTM methods, the windows are prescreened first by the proposed hypothesis test in a mixture-eliminating procedure. Based on our self-adjusted strategy to determine the test threshold, 
the final acceptance maps for the two scenes are shown in Fig. 15 and Fig. 16 when the non-uniformity ratio is set to be lower than $10 \%$. Only the windows labeled as uniform here will be used for global ENL estimation. The overall distributions of ENL estimates obtained with different methods are shown in different panels of Fig. 17 and Fig. 18, with dashed lines marking the mode values. The achieved global ENL estimates are all recorded in Table. II. The reference values listed there are obtained by estimating the ENL with the ML algorithm on manually selected homogeneous regions in the corresponding image. As we notice, all the estimates are lower than the nominal number of looks. This is because the averaged samples in multi-looking process that are correlated and correspond to less independent samples represented by ENL.

For the first data set, the windows along the bridge and the coastline are not used towards the final ENL estimate, as shown in Fig. 15. From the overall distributions in Fig. 17, we notice that the left tails of the MEML and the MEDTM distributions have been clearly shrunken comparing with those of the ML and DTM distributions, respectively. Especially, the secondary hump located between 3 and 5 in the ML distribution curve has been completely removed in the MEML curve. But as the homogeneous areas dominate, the nonuniform windows do not have a big effect on the mode values of the overall distributions and the global ENL estimate of the ML and DTM methods are already closed to the reference value. In this case the mixture elimination is strictly not necessary and the final results are only slightly altered with the MEML and MEDTM methods.

The effect of the proposed mixture-eliminating process becomes more prominent for the complex San Francisco image. As shown in Fig. 16, the urban area with man-made structures and the coastline area are labeled as non-uniform, as we expect. After discarding the non-uniform windows, the mode values of MEML and MEDTM estimate distributions are both shifted to the right, which get much closer to the reference value than those of ML and DTM.

As noted previously, the ML estimator produces low ENL

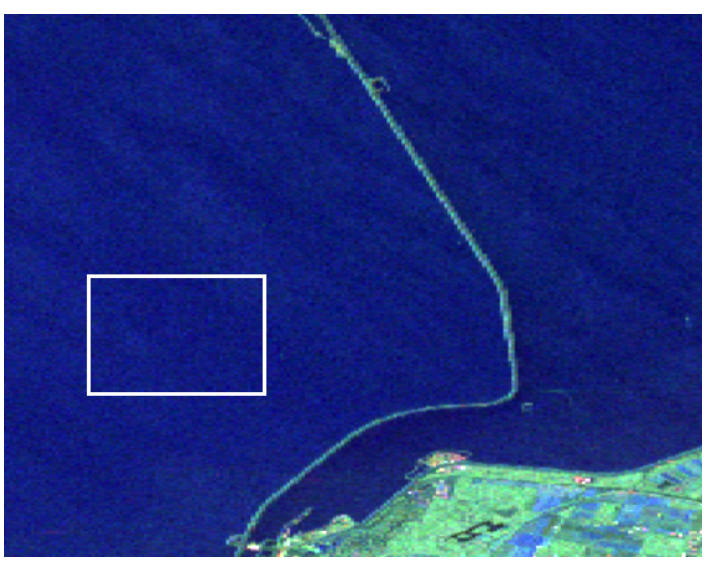

Fig. 13: Pauli decomposition composite image of the Flevoland data set, the region within the white box is used to obtain the reference ENL value. estimates in heterogeneous windows. Hence, these underestimated values and the estimates from homogeneous windows form different peaks in the overall distributions, which explains the multimodality of the distribution obtained by both the ML and MEML methods. As the majority of the sliding windows in the original image contain a mixture of different classes or cover textured regions, the estimates from homogeneous windows are overwhelmed in the ML distribution. With the MEML method, most non-uniform windows are excluded by the designed hypothesis test. Although some of the accepted windows still cover textured areas like vegetated hills, the windows occupied by fully developed speckle become dominant. Therefore, an accurate global ENL estimate is obtained with the MEML method. Still we have to admit that the estimation accuracy of the MEML estimator depends on the dominance of homogeneous windows among those detected as uniform.

For the texture-invariant DTM estimator, the mixtureeliminating process actually removes the windows which contain multiple classes and do not follow the product model that the invariance property is based on. Therefore, the MEDTM method addresses both the mixture of different classes and texture variance within a single-class region, the two main sources of heterogeneity affecting the ENL estimation. As a result, the MEDTM estimates form a unimodal distribution, as shown in the bottom panel in Fig. 18. However, the higher variance of the DTM estimator causes the mode value to deviate from the actual ENL value when samples are limited, as in this case.

There are drawbacks to both adopted estimators. Therefore, a more reliable estimator, which is both texture-invariant and low-variance, needs to be further investigated.

\section{CONCLUSION}

We have proposed an unsupervised ENL estimation method, which maintains the automaticity of the small-sample estimation strategy by applying ENL estimator to sliding windows over an arbitrary PolSAR image, but adopts a mixtureeliminating procedure to reduce the effect of non-uniform win-

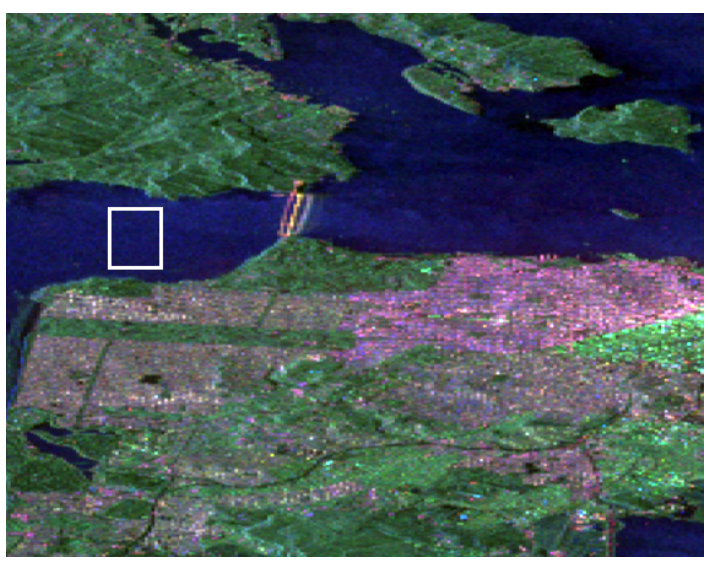

Fig. 14: Pauli decomposition composite image of the San Francisco data set, the region within the white box is used to obtain the reference ENL value. 


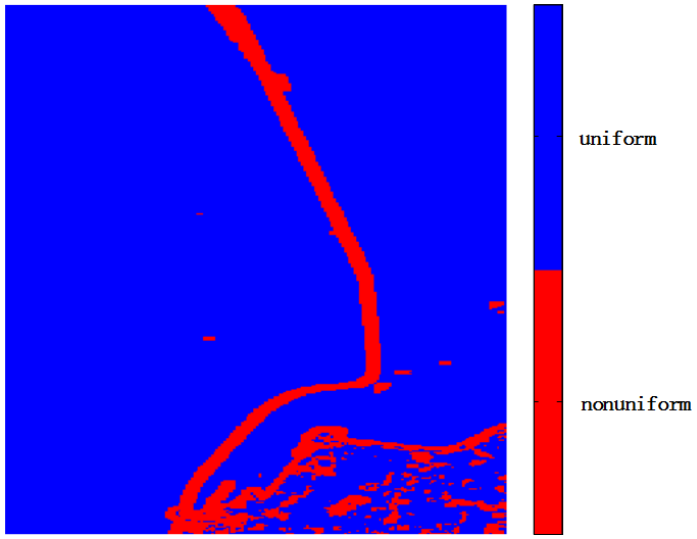

Fig. 15: Final acceptance map of the Flevoland data set

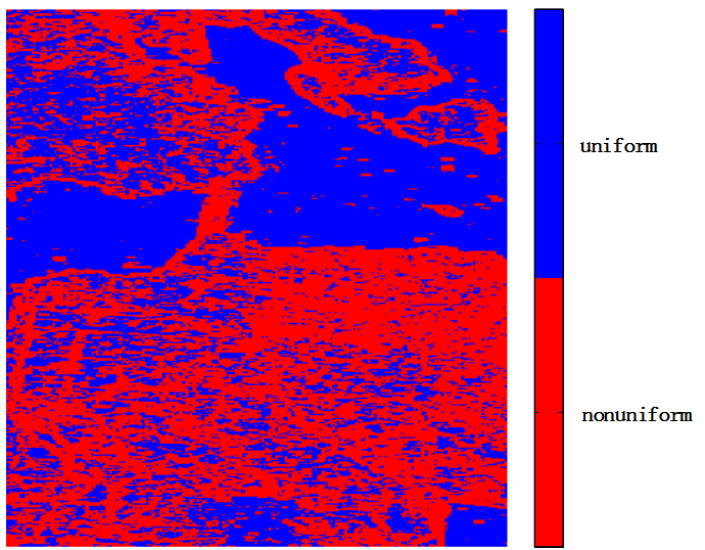

Fig. 16: Final acceptance map of the San Francisco data set

dows on the global estimate. The ME procedure distinguishes the uniform and non-uniform windows by implementing a hypothesis test with adaptive thresholds based on the empirical distribution of the derived test statistic.

This paper demonstrates the feasibility of the used test statistic through the theoretical derivation and the simulation experiment. Assessment of the statistical properties shows that the sampling distribution of the derived test statistic for uniform windows is symmetric and zero-mean, while that for non-uniform window biases towards one side of zero value. Then we use the rescaled overlapping region of the overall distributions of two test statistics with reversed channel order to approximate the distribution under the hypothesis corresponding to uniformity. The experiment with simulated data, for which locations of uniform windows are known, indicates the effectiveness of the mixture-eliminating procedure. The resulting acceptance map confirms that the proposed approach

TABLE II: The global ENL estimates of different methods for the two data sets. The NL refers to the nominal number of looks. The reference values are obtained by applying ML algorithm to manually selected homogeneous regions.

\begin{tabular}{lcccccc}
\hline Method & NL & ML & MEML & DTM & MEDTM & reference \\
\hline Flevoland & 25 & 12.1 & 12.3 & 11.2 & 11.5 & 12.4 \\
San Francisco & 25 & 4.9 & 11.4 & 5.2 & 9.9 & 11.7 \\
\hline
\end{tabular}

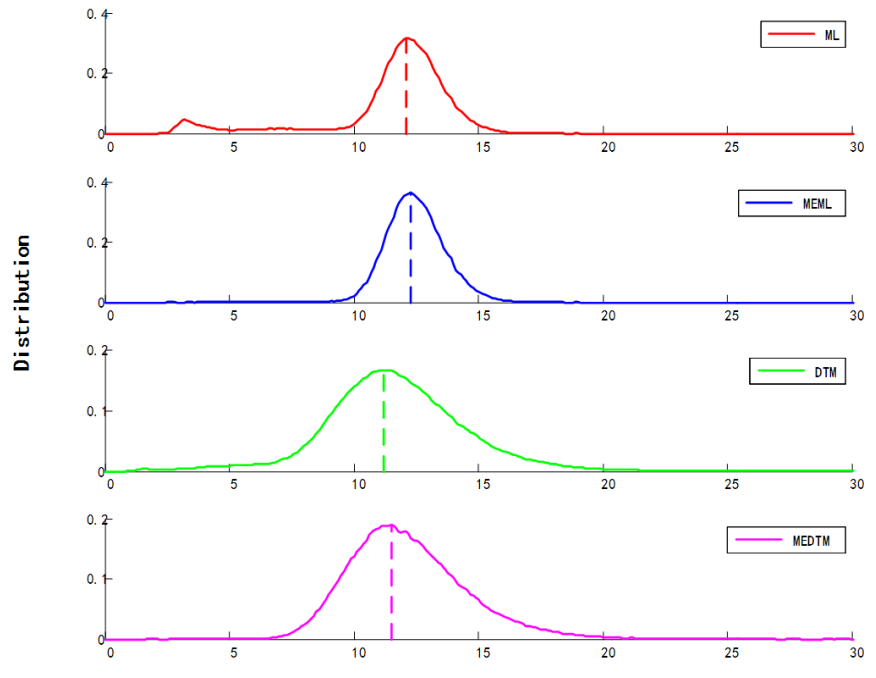

ENL

Fig. 17: Distribution estimates for the ML, MEML, DTM, MEDTM method from the Flevoland data set

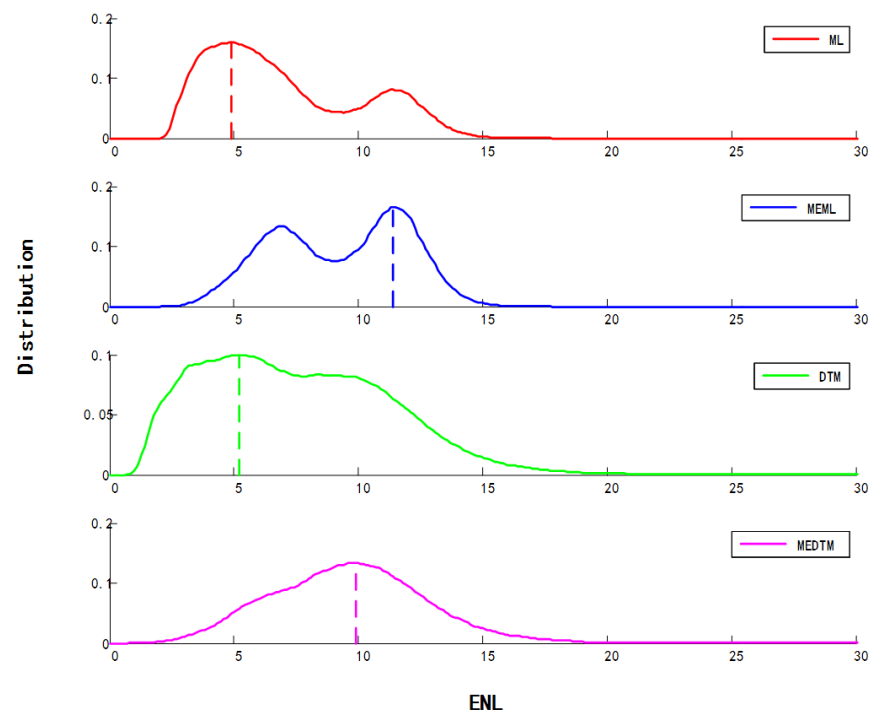

Fig. 18: Distribution estimates for the ML, MEML, DTM, MEDTM method from the San Francisco data set

can achieve performance very close to the predefined label map based on ground truth.

Finally, we examined the robustness of the proposed ENL estimation strategy with two real PolSAR images of varying complexities. The new approach has been compared with the original small-sample estimation method where the ENL estimator, ML or DTM, is directly applied to every window. It is shown that the mixture-eliminating procedure is necessary, especially for complicated scenes, to ensure extraction of a more reliable ENL estimate from the overall distribution of small sample estimates. However, as both of the adopted estimators have drawbacks, a more comprehensive estimator is still sought. 


\section{APPENDIX A}

\section{PROOF OF ANALYTICAL TEST STATISTIC EXPRESSION IN} NON-UNIFORM WINDOWS

Suppose a window contains two different classes, class 1 and class 2 , whose texture variable is $T_{1}$ and $T_{2}$, respectively. In given polarimetric channels, $a$ and $b$, the log-cumulants of the two classes can be formulated as:

$$
\begin{aligned}
& E\left\{\ln I_{a}^{(1)}\right\}=\ln E\left\{I_{a}^{(1)}\right\}+D(L)+f\left(T_{1}\right), \\
& E\left\{\ln I_{b}^{(1)}\right\}=\ln E\left\{I_{b}^{(1)}\right\}+D(L)+f\left(T_{1}\right), \\
& E\left\{\ln I_{a}^{(2)}\right\}=\ln E\left\{I_{a}^{(2)}\right\}+D(L)+f\left(T_{2}\right), \\
& E\left\{\ln I_{b}^{(2)}\right\}=\ln E\left\{I_{b}^{(2)}\right\}+D(L)+f\left(T_{2}\right),
\end{aligned}
$$

Here, we use the $I_{m}^{(n)}$ to represent the intensity of class $n$ in channel $m$. Then the intensity ratio between class 1 and 2 in channel $m$ can be denoted by $R_{m}=E\left\{I_{m}^{(1)}\right\} / E\left\{I_{m}^{(2)}\right\}$. The prior probabilities of these two classes are assumed as $p_{1}$ and $p_{2}$, respectively, which follows

$$
p_{1}+p_{2}=1 .
$$

Then the logarithmic mean value of all the samples in the window can be written in the terms of the logarithmic mean values of class 1 and class 2 , that is,

$$
\begin{aligned}
& E\left\{\ln I_{a}\right\}=p_{1} E\left\{\ln I_{a}^{(1)}\right\}+p_{2} E\left\{\ln I_{a}^{(2)}\right\}, \\
& E\left\{\ln I_{b}\right\}=p_{1} E\left\{\ln I_{b}^{(1)}\right\}+p_{2} E\left\{\ln I_{b}^{(2)}\right\} .
\end{aligned}
$$

Therefore,

$$
\begin{aligned}
& {\left[\ln E\left\{I_{a}\right\}-E\left\{\ln I_{a}\right\}\right]-\left[\ln E\left\{I_{b}\right\}-E\left\{\ln I_{b}\right\}\right]} \\
& =\left[\ln E\left\{I_{a}\right\}-\ln E\left\{I_{b}\right\}\right]-\left[E\left\{\ln I_{a}\right\}-E\left\{\ln I_{b}\right\}\right] \\
& =\left[\ln E\left\{I_{a}\right\}-\ln E\left\{I_{b}\right\}\right]-\left[p_{1} E\left\{\ln I_{a}^{(1)}\right\}+p_{2} E\left\{\ln I_{a}^{(2)}\right\}\right. \\
& \left.\quad-p_{1} E\left\{\ln I_{b}^{(1)}\right\}-p_{2} E\left\{\ln I_{b}^{(2)}\right\}\right] \\
& =\left[\ln E\left\{I_{a}\right\}-\ln E\left\{I_{b}\right\}\right]-\left[p_{1} \ln E\left\{I_{a}^{(1)}\right\}+p_{2} \ln E\left\{I_{a}^{(2)}\right\}\right. \\
& \left.\quad-p_{1} \ln E\left\{I_{b}^{(1)}\right\}-p_{2} \ln E\left\{I_{b}^{(2)}\right\}\right] \\
& =\left[\ln E\left\{I_{a}\right\}-p_{1} \ln E\left\{I_{a}^{(1)}\right\}-p_{2} \ln E\left\{I_{a}^{(2)}\right\}\right]- \\
& =\ln \frac{\left[\ln E\left\{I_{b}\right\}-p_{1} \ln E\left\{I_{b}^{(1)}\right\}-p_{2} \ln E\left\{I_{b}^{(2)}\right\}\right]}{E\left\{I_{a}^{(1)}\right\}^{p_{1}} E\left\{I_{a}^{(2)}\right\} p_{2}}-\ln \frac{E\left\{I_{b}\right\}}{E\left\{I_{b}^{(1)}\right\}^{p_{1}} E\left\{I_{b}^{(2)}\right\} p^{p_{2}}} \\
& =\ln \frac{p_{1} E\left\{I_{a}^{(1)}\right\}+p_{2} E\left\{I_{a}^{(2)}\right\}}{E\left\{I_{a}^{(1)}\right\}^{p_{1}} E\left\{I_{a}^{(2)}\right\}^{p_{2}}}-\ln \frac{p_{1} E\left\{I_{b}^{(1)}\right\}+p_{2} E\left\{I_{b}^{(2)}\right\}}{E\left\{I_{b}^{(1)}\right\} p_{1} E\left\{I_{b}^{(2)}\right\} p_{2}} \\
& =\ln \frac{\left(p_{1} E\left\{I_{a}^{(1)}\right\}+p_{2} E\left\{I_{a}^{(2)}\right\}\right)\left(E\left\{I_{b}^{(1)}\right\}^{p_{1}} E\left\{I_{b}^{(2)}\right\}^{p_{2}}\right)}{\left(E\left\{I_{a}^{(1)}\right\}^{p_{1}} E\left\{I_{a}^{(2)}\right\}^{p_{2}}\right)\left(p_{1} E\left\{I_{b}^{(1)}\right\}+p_{2} E\left\{I_{b}^{(2)}\right\}\right)} \\
& =\ln \frac{\left(p_{1} E\left\{I_{a}^{(1)}\right\}+p_{2} E\left\{I_{a}^{(2)}\right\}\right)\left(E\left\{I_{b}^{(1)}\right\}^{p_{1}} E\left\{I_{b}^{(2)}\right\}^{p_{2}}\right)}{\left(p_{1} E\left\{I_{b}^{(1)}\right\}+p_{2} E\left\{I_{b}^{(2)}\right\}\right)\left(E\left\{I_{a}^{(1)}\right\}^{p_{1}} E\left\{I_{a}^{(2)}\right\}^{p_{2}}\right)} \\
& =\ln \frac{p_{1} R_{a}^{p_{2}}+p_{2} R_{a}^{-p_{1}}}{p_{1} R_{b}^{p_{2}}+p_{2} R_{b}^{-p_{1}}} \\
& =\ln \frac{p_{1} R_{a}^{1-p_{1}}+\left(1-p_{1}\right) R_{a}^{-p_{1}}}{p_{1} R_{b}^{1-p_{1}}+\left(1-p_{1}\right) R_{b}^{-p_{1}}} \\
& =\ln \frac{g\left(R_{a}\right)}{g\left(R_{b}\right)}
\end{aligned}
$$

where the function $g(\cdot)$ will be studied shortly. Actually a uniform window is the special case when $p_{1}=0$ or 1 , and thus

$$
\left[\ln E\left\{I_{a}\right\}-E\left\{\ln I_{a}\right\}\right]-\left[\ln E\left\{I_{b}\right\}-E\left\{\ln I_{b}\right\}\right]=0 .
$$

For a non-uniform window where $0<p_{1}<1$, and where there exists at least two channels $a$ and $b$, such that $R_{a} \neq R_{b}$, then the test statistic can be written in the terms of the function

$$
g(r)=p r^{1-p}+(1-p) r^{-p}, 0<p<1, r>0
$$

whose derivative is

$$
\begin{aligned}
\frac{d g(r)}{d r} & =p(1-p)^{-p}-p(1-p) r^{-p-1} \\
& =p(1-p) r^{-p}\left(1-r^{-1}\right)
\end{aligned}
$$

When $0<r<1, g(r)$ is monotonically decreasing. When $r>1, g(r)$ is monotonically increasing, and $g(1)=1$. Therefore, only when $R_{a}$ and $R_{b}$ happen to be two different roots of $p_{1} r^{1-p_{1}}+\left(1-p_{1}\right) r^{-p_{1}}=$ Const ( Const is a constant larger than 1), then eq. (51) could hold for non-uniform window. However, such a special case just exists in a mathematical sense. In most real data, it would not appear,that is, for non-uniform windows, the statistic $\left[\ln E\left\{I_{a}\right\}-E\left\{\ln I_{a}\right\}\right]-\left[\ln E\left\{I_{b}\right\}-E\left\{\ln I_{b}\right\}\right]$ will in practice be unequal to 0 with high probability.

\section{REFERENCES}

[1] A. P. Doulgeris, "An Automatic U Distribution and Markov Random Field Segmentation Algorithm for PolSAR Images," IEEE Transactions on Geoscience and Remote Sensing, vol. 53, pp. 1819-1827, 2015.

[2] A. P. Doulgeris, S. N. Anfinsen, and T. Eltoft, "Automated Non-Gaussian Clustering of Polarimetric Synthetic Aperture Radar Images," IEEE Transactions on Geoscience and Remote Sensing, vol. 49, pp. 3665-3676, 2011.

[3] A. C. Frery, A. H. Correia, and C. d. C. Freitas, "Classifying Multifrequency Fully Polarimetric Imagery With Multiple Sources of Statistical Evidence and Contextual Information," IEEE Transactions on Geoscience and Remote Sensing, vol. 45, pp. 3098-3109, 2007.

[4] K. Conradsen, A. A. Nielsen, J. Schou, and H. Skriver, "A test statistic in the complex Wishart distribution and its application to change detection in polarimetric SAR data," IEEE Transactions on Geoscience and Remote Sensing, vol. 41, pp. 4-19, 2003.

[5] C. Oliver and S. Quegan, Understanding synthetic aperture radar images: Raleigh, 2004.

[6] J. S. Lee and K. Hoppel, "Noise Modeling and Estimation of RemotelySensed Images," in Geoscience and Remote Sensing Symposium, IGARSS. Vancouver, BC, Canada, Jul. 1989, vol.2, pp. 1005-1008.

[7] J.-S. Lee, K. Hoppel, and S. A. Mango, "Unsupervised estimation of speckle noise in radar images," International Journal of Imaging Systems and Technology, vol. 4, pp. 298-305, 1992.

[8] S. Foucher, J. M. Boucher, and G. B. Benie, "Maximum likelihood estimation of the number of looks in SAR images," in Microwaves, Radar and Wireless Communications. 2000. MIKON-2000. 13th International Conference on, 2000, pp. 657-660 vol.2.

[9] S. N. Anfinsen, A. P. Doulgeris, and T. Eltoft, "Estimation of the Equivalent Number of Looks in Polarimetric Synthetic Aperture Radar Imagery," IEEE Transactions on Geoscience and Remote Sensing, vol. 47, pp. 3795-3809, 2009.

[10] T. Liu, H.-g. Cui, Z.-m. Xi, and J. Gao, "Texture-Invariant Estimation of Equivalent Number of Looks Based on Trace Moments in Polarimetric Radar Imagery," Geoscience and Remote Sensing Letters, IEEE, vol. 11, pp. 1129-1133, 2014.

[11] D. Hu, S. N. Anfinsen, D. Tao,, and X. Qiu, "Investigation Of Variations In The Equivalent Number Of Looks For Polarimetric Channels," in 7th International Workshop on Science and Applications of SAR Polarimetry and Polarimetric Interferometry, ESA POLinSAR 2015, 2015. 
[12] J. S. Lee and E. Potter, Polarimetric Radar Imaging From Basic to Application. New York: CSC Press, 2009.

[13] C. C. Freitas, A. C. Frery, and A. H. Correia, "The Polarimetric G Distribution for SAR Data Analysis," Environmetrics, vol. 16, pp. 1331, 2005.

[14] R. Touzi, W. M. Boerner, J. S. Lee, and E. Lueneburg, "A review of polarimetry in the context of synthetic aperture radar: concepts and information extraction," Canadian Journal of Remote Sensing, vol. 30, pp. 380-407, 2004/01/01 2004.

[15] J. S. Lee, K. W. Hoppel, S. A. Mango, and A. R. Miller, "Intensity and phase statistics of multilook polarimetric and interferometric SAR imagery," IEEE Transactions on Geoscience and Remote Sensing, vol. 32, pp. 1017-1028, 1994.

[16] N. R. Goodman, "Statistical Analysis Based on a Certain Multivariate Complex Gaussian Distribution (An Introduction)," Ann. Math. Stat.. pp. 152-177, 1963/03 1963.

[17] J.-M. Nicolas,'Introduction aux statistique de deuxième espèce: Application des logs-moments et des logs-cumulants à lanalyse des lois d'images radar," Traitement du Signal, vol. 19, no. 3, pp. 139-167, 2002, in French, English translation in [19].

[18] J.-M. Nicolas,"Application de la transformée de Mellin : étude des lois statistiques de l'imagerie cohérente," Ecole Nationale Supérieure des Télécommunications, Paris, France, Tech. Rep. 2006D010, 2006, in French.

[19] S. N. Anfinsen, "Statistical analysis of multilook polarimetric radar images with the Mellin transform," Ph.D. dissertation, University of Troms, Troms, Norway, 2010.

[20] J. S. Lee, D. L. Schuler, R. H. Lang, and K. J. Ranson, "K-distribution for multi-look processed polarimetric SAR imagery," in Geoscience and Remote Sensing Symposium, 1994. IGARSS '94. Surface and Atmospheric Remote Sensing: Technologies, Data Analysis and Interpretation., International, 1994, pp. 2179-2181 vol.4.

[21] L. Bombrun and J. M. Beaulieu, "Fisher distribution for texture modeling of polarimetric SAR data," IEEE Geoscience and Remote Sensing Letters, vol. 5, pp. 512-516, Jul 2008.

[22] S. N. Anfinsen and T. Eltoft, "Application of the Matrix-Variate Mellin Transform to Analysis of Polarimetric Radar Images," IEEE Transactions on Geoscience and Remote Sensing, vol. 49, pp. 2281-2295, 2011.

[23] B. W. Silverman, Density estimation for statistics and data analysis vol. 26: Chapman \& Hall/CRC, 1998.

[24] M. P. Wand and M. C. Jones, Kernel smoothing vol. 60: Chapman \& Hall, 1995.

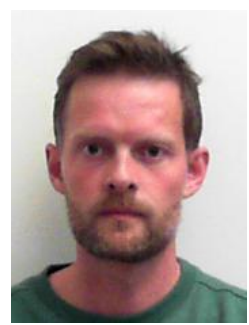

Stian Normann Anfinsen (S06,M10) received the Cand. Mag. (B.Sc.), Cand. Scient. (M.Phil.) and $\mathrm{Ph} . \mathrm{D}$. degrees in physics from the University of Troms, Troms, Norway in 1997, 2000 and 2010, respectively. He received the M.Sc. in Communications, Control and Digital Signal Processing with distinction from the University of Strathclyde, Glasgow, U.K. in 1998. He was with satellite ground station system provider Kongsberg Spacetec in Troms from 2001 to 2005 . He then moved to the University of Troms The Arctic University of Norway, Department of Physics and Technology, where he is currently an associate professor at the Machine Learning Group. His research in earth observation and remote sensing has focused on statistical modelling, parameter estimation, classification, change detection and target detection in polarimetric synthetic aperture radar images. His current interests also include information extraction from multimodal images and deep learning. He has been recognized as the 2012 Best Reviewer of the IEEE Journal of Selected Topics in Applied Earth Observations and Remote Sensing.

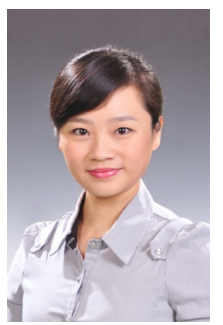

Xiaolan Qiu received the B.S. degree in electronic engineering and information science from the University of Science and Technology of China (USTC) in 2004 and the Doctoral degree in signal and information processing from the Graduate University of Chinese Academy of Sciences (GUCAS) in 2009. Since then, she has worked in the Institute of Electronics, Chinese Academy of Sciences (IECAS). Between May to November 2011, she was a Visiting Scholar with the University of Siegen in Germany. Her main research topics includes SAR imaging and geo-correction, SAR simulation, and SAR image interpretation.

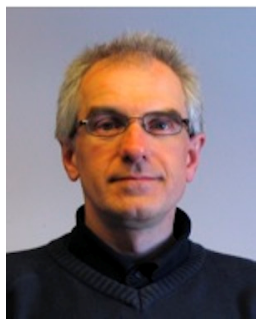

Anthony P. Doulgeris (S06M12) received the B.Sc. degree in physics from The Australian National University, Canberra, Australia, in 1988, the M.Sc. degree and the Ph.D. degree in physics from the Department of Physics and Technology, UiT The Arctic University of Norway, Troms $\varnothing$, Norway, in 2006, and 2011, respectively.

He joined the Department of Physics and Technology at the UiT The Arctic University of Norway, in 2007 and is currently an associate professor in applied Earth observation. His research interests focus on investigating remote sensing, pattern recognition and multidimensional statistical modelling, in particular with polarimetric synthetic aperture radar images.

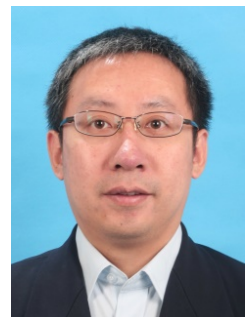

Bin Lei received the B.Sc. degree in electrical engineering from The Tsinghua University, Beijing, China, in 2000, the M.Sc. degree and the Ph.D. degree in signal and information processing from the Institute of Electronic, Chinese Academy of Science, Beijing, China, in 2003, and 2014, respectively.

$\mathrm{He}$ is currently a professor and the director in Key Laboratory of Technology in Geo-spatial Information Processing and Application System, Institute of Electronics, Chinese Academy of Sciences. His research interests focus on investigating remote sensing data processing and system applications. 